\title{
¿LA CRISIS DE LA BIODIVERSIDAD ES LA CRISIS DE LA TAXONOMÍA?
}

\author{
José LUIS VILLASEÑOR \\ Departamento de Botánica, Instituto de Biología, Universidad Nacional Autónoma de México, México, D. F., México \\ vrios@ib.unam.mx
}

\begin{abstract}
Resumen: El compromiso de integrar una lista florística que plasme el conocimiento de la diversidad vegetal a corto plazo, solamente será alcanzado con un cuerpo sólido de taxónomos especialistas, el cual, en lugar de fortalecerse, más bien parece estar en decaimiento. Los objetivos de este trabajo son discutir el papel que han jugado los taxónomos mexicanos en el conocimiento de la flora de México y cuál es el capital humano con que se cuenta en la actualidad, así como analizar el conocimiento que se tiene actualmente sobre la flora de México y su relevancia en las estrategias de conservación de la biodiversidad. Se proponen además, estudios novedosos que podrían complementar el conocimiento taxonómico para lograr definir las zonas más importantes de riqueza y endemicidad (hotspots) que complementen o actualicen nuestro Sistema Nacional de Áreas Naturales Protegidas. Los principales resultados señalan que la tasa de descripción de especies nuevas todavía muestra un sesgo importante en el número total de especies en la flora mexicana, ya que cada año se adiciona un número importante, ya sea como especies o como registros nuevos. Gran parte del descubrimiento de la diversidad faltante es obtenido con la revisión especializada del estudio de las colecciones científicas, aunque el número de taxónomos especialistas ha sufrido una disminución considerable. Se discuten algunas líneas emergentes de estudio que han ayudado a definir mejores estrategias de búsqueda y análisis de la biodiversidad, que seguramente facilitarán el trabajo de exploración e investigación de este componente desconocido de la diversidad vegetal del país. Se propone que, evitando la crisis que enfrenta actualmente la taxonomía, se minimizará de manera importante la crisis de la biodiversidad y de otras áreas de investigación afines. Desafortunadamente, el problema de la desaparición de los taxónomos en el mundo (y México no es la excepción), se debe en gran parte a los mecanismos de evaluación de la producción académica, a los ingresos obtenidos mediante recursos extraordinarios (subsidios a proyectos), y al factor de impacto de las revistas donde se publican los resultados. Se debe resolver esto para evitar considerarlos una especie en extinción.
\end{abstract}

Palabras clave: biomas, colecciones científicas, diversidad vegetal, florística, hotspots, México.

\begin{abstract}
We will achieve the commitment of integrating a floristic list that reflects the knowledge of the Mexican plant diversity in the short term only with a solid body of plant taxonomists. Unfortunately, it seems that such body of specialists rather than strengthening is decaying. The goals of this paper are to discuss the role played by the Mexican taxonomists in the knowledge of the flora of Mexico and its current human capital, and to analyze the updated floristic knowledge and its relevance for the biodiversity conservation strategies. Novel approaches would help to complement our taxonomic knowledge by defining additional areas of richness and endemism (hotspots) that supplement or make more efficient our national system of natural protected areas. The main results point out that the rate of description of new species suggests there is still a significant bias in knowing the total number of species in the Mexican flora, but each year an important number of them, either as new species or new records, are added. Much of the discovery of this missing diversity is achieved with specialized review of the scientific collections, although the number of taxonomists has dramatically decreased. Some emerging lines of research that help to define better strategies for searching and analyzing biodiversity are discussed; they will surely facilitate the work of exploration and investigation of this unknown component of plant diversity of the country. It is proposed that avoiding the actual crisis of taxonomy we will minimize the biodiversity crisis. Unfortunately the disappearance of taxonomist in the world (and Mexico is not an exception) is due mostly to the actual way to evaluating the academic productivity, to the difficulty of obtaining grant resources for taxonomic studies, as well as to the low impact factor of journals were their results are published. Changes are necessary to avoid considering taxonomists an endangered species.
\end{abstract}

Keywords: biomes, floristics, hotspots, Mexico, plant diversity, scientific collections.

$\mathbf{H}$ ace ya dos décadas, un reporte al gobierno británico, encabezado por J.R. Krebs (1992), plasmó una serie de recomendaciones en torno a la necesidad de encauzar un ambicioso programa que consolidara los estudios taxonómi- cos para lograr un mejor conocimiento de la biodiversidad. Poco después, un consorcio de instituciones norteamericanas propuso lo que llamó la Systematics Agenda 2000, destacando la enorme distancia que todavía existía entre la 
diversidad registrada hasta la fecha y la que permanecía aún desconocida (Systematics Agenda, 1994). Uno de los principales objetivos de ambos documentos fue lograr la meta de descubrir, describir e inventariar la riqueza de especies existente en el mundo. En 2008, La Comisión Nacional para el Conocimiento y Uso de la Biodiversidad (CONABIO) y otras agencias gubernamentales mexicanas establecieron la Estrategia Mexicana para la Conservación Vegetal (CONABIO et al., 2008; CONABIO, 2012), donde su Meta 1 menciona: "contar con la lista completa e integrada de especies vegetales mexicanas y sus categorías infraespecíficas”. La visión planteada es que para 2030 se logre "incrementar e integrar el conocimiento de la diversidad mexicana...”. Dichos documentos subrayan la necesidad de contar con un buen conocimiento taxonómico de la flora de México, consolidado por un adecuado número de taxónomos participando en su generación.

Estamos a tres lustros de la fecha propuesta (2030) como meta para contar con el adecuado conocimiento científico de nuestra diversidad vegetal y a siete años de que fue instituida dicha estrategia nacional (2008). Sería interesante hacer una evaluación del conocimiento taxonómico y del capital humano especializado en el tema, con el fin de saber cuánto se ha avanzado y la posibilidad de alcanzar esta importante

Cuadro 1. Número de especies nuevas (incluyendo taxa subespecíficos), descritas para la flora de México y combinaciones taxonómicas realizadas desde 1753 a la fecha.

\begin{tabular}{cccc}
\hline Periodo & $\begin{array}{c}\text { Especies } \\
\text { nuevas }\end{array}$ & $\begin{array}{c}\text { Combinaciones } \\
\text { taxonómicas }\end{array}$ & $\begin{array}{c}\text { Total } \\
\text { especies }\end{array}$ \\
\hline $1753-1800$ & 938 & 60 & 998 \\
$1801-1810$ & 255 & 86 & 341 \\
$1811-1820$ & 427 & 87 & 514 \\
$1821-1830$ & 546 & 203 & 749 \\
$1831-1840$ & 803 & 215 & 1,018 \\
$1841-1850$ & 901 & 180 & 1081 \\
$1851-1850$ & 741 & 178 & 919 \\
$1861-1870$ & 381 & 163 & 544 \\
$1871-1880$ & 492 & 151 & 643 \\
$1881-1890$ & 868 & 281 & 1,149 \\
$1891-1900$ & 968 & 380 & 1,348 \\
$1901-1910$ & 908 & 322 & 1,230 \\
$1911-1920$ & 713 & 357 & 1,070 \\
$1921-1930$ & 725 & 419 & 1,144 \\
$1931-1940$ & 947 & 364 & 1,311 \\
$1941-1950$ & 658 & 323 & 981 \\
$1951-1960$ & 363 & 168 & 531 \\
$1961-1970$ & 587 & 421 & 1,008 \\
$1971-1980$ & 898 & 712 & 1,610 \\
$1981-1990$ & 1,143 & 670 & 1,813 \\
$1991-2000$ & 1,195 & 658 & 1,853 \\
$2001-2014$ & 1,077 & 890 & 1,967 \\
Total & 16,534 & 7,288 & 23,822 \\
\hline
\end{tabular}

tarea. Por ejemplo, los valores mínimos propuestos sobre la riqueza florística del país oscilan entre 21,000 y 23,000 especies, cifras que no han variado demasiado, no así las estimaciones sobre los valores máximos de diversidad (2630,000 especies), que todavía muestran gran incertidumbre (Rzedowski, 1991; Toledo, 1994; Villaseñor, 2003; EspejoSerna et al., 2004; Llorente-Bousquets y Ocegueda, 2008; Villaseñor y Ortiz, 2014).

El cuadro 1 muestra las descripciones de especies nuevas y combinaciones taxonómicas de los nombres de plantas vasculares que son aceptados en la actualidad para México, distribuidos por decenios. La fecha inicial (1753) corresponde a la publicación de la obra de Linneo, que el Código Internacional de Nomenclatura Botánica (McNeill et al., 2006) considera como el parteaguas de la nomenclatura moderna. El número promedio de nombres aceptados, tanto nuevos como producto de combinaciones taxonómicas por año, es de 1,083. Sin embargo, en los últimos 40 años la proporción de nombres nuevos rebasa considerablemente esta media, indicando un repunte importante de la actividad taxonómica en el país en dicho periodo.

Un aspecto destacable del cuadro 1 es la cantidad de combinaciones taxonómicas llevadas a cabo en las especies de la flora de México. Considerando el total de especies y taxa subespecíficos $(23,822)$ reconocidos actualmente, 7,288 han sufrido cambios nomenclaturales con respecto a cómo fueron descritas originalmente; en otras palabras, $31 \%$ de la flora de México es reconocida con otros nombres que siguen las reglas internacionales de nomenclatura y que representan reajustes en la taxonomía y sistemática de los grupos vegetales a que pertenecen. Muchas de las combinaciones no han sido aplicadas en las publicaciones que discuten aspectos de nuestra flora, o más desafortunadamente, se utilizan en conjunto con sus basiónimos; es decir, los nombres originales con que fueron descritas las especies. Esta duplicación sobrestima la lista de especies reconocidas en la flora de México, ya que muchas de ellas deben de ser reconocidas como sinónimos.

La figura 1 muestra los datos del cuadro 1 ordenados por fecha de publicación, con el fin de evaluar la tasa de descripciones o combinaciones nuevas de las especies actualmente reconocidas como aceptadas. Las pendientes observadas en la gráfica, tanto para el total de especies nuevas como el de combinaciones nomenclaturales, no han alcanzado su asíntota; por lo tanto, es de esperar que la descripción de especies nuevas y de rearreglos taxonómicos que derivan en las nuevas combinaciones nomenclaturales continúe a un ritmo parecido al que se ha observado a lo largo del tiempo, siempre y cuando se cuente con el número de taxónomos suficientes. Una de las grandes preocupaciones a nivel mundial, es la carencia cada vez mayor de especialistas en el campo de la taxonomía, no obstante su papel crucial en la generación de conocimiento básico para otras ramas de la ciencia. 


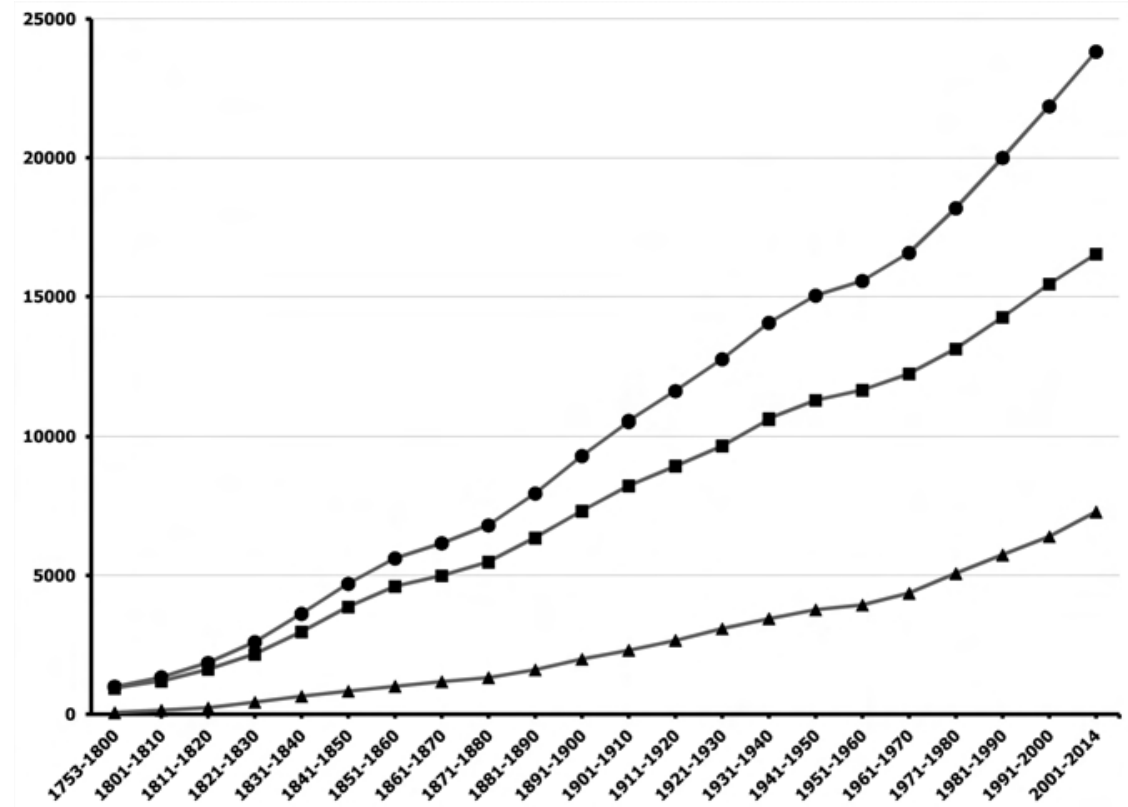

Figura 1. Tasa de descripción de especies nuevas (incluyendo taxa subespecíficos) y combinaciones taxonómicas en la flora de México, realizadas desde 1753 a la fecha. La línea con círculos muestra la tendencia con el total de especies, la línea con cuadros las especies aceptadas sin cambios nomenclaturales y la línea con triángulos los cambios nomenclaturales aceptados.

Por ejemplo, una preocupación actual entre los interesados y estudiosos de la naturaleza, es la actual tasa de extinción de las especies. De hecho, ya se discute que estamos enfrentando un nuevo periodo de extinción masiva de especies (llamada la sexta extinción masiva; Leakey y Lewin, 1996), y que muchas de las especies que actualmente se están extinguiendo pasarán al componente desconocido de la biodiversidad, que ni siquiera se podrá tener una idea del papel que desempeñan en los ecosistemas que habitan actualmente.

Por otra parte, el hombre obtiene una amplia gama de bienes materiales de la biodiversidad, ya sea de tipo alimenticio, vestido, materiales para la construcción o de leña. Incluso, muchos de los medicamentos que se usan actualmente provienen de unas cuantas docenas de plantas, animales o microorganismos, todos ellos especies taxonómicamente bien definidas y circunscritas. Se estima que más del $90 \%$ de las especies de plantas con flores conocidas actualmente nunca han sido estudiadas para conocer posibles productos naturales de importancia medicinal (von Reis Altschul, 1977). Sin embargo, se ha documentado ampliamente que las plantas no pueden evadir directamente a sus depredadores, al ser organismos sésiles, por lo que han desarrollado defensas químicas (metabolitos secundarios), que las han defendido y permitido mantenerse como ejemplos vivientes de la biodiversidad del planeta. Los metabolitos secundarios han surgido mediante un proceso paulatino, desarrollado por las especies a lo largo de millones de años de evolución. En la actualidad, ni los más sofisticados laboratorios de química (Firn, 2010), capaces de generar miles de compuestos, han podido alcanzar lo que la naturaleza aporta al hombre, ni avanzar de manera importante para disminuir su dependencia a medicamentos de origen natural. Por tal motivo, la bioprospección, es decir, la búsqueda en la naturaleza de productos naturales que sean útiles al ser humano, es una de las actividades más importantes para subsanar la deficiencia de medicamentos para muchos de nuestros padecimientos. ¿Pero dónde buscar, cuando nuestro desconocimiento acerca de la biodiversidad es tan grande y la tasa de destrucción de los ecosistemas ocurre a una velocidad mayor a la que se requiere para conocerla?

Se está acelerando la extinción de muchas especies, a pesar de las numerosas estrategias propuestas para su conservación por la Biología de la Conservación (VaneWright et al., 1991; Pimm et al., 1995; Margules y Pressey, 2000), estrategias que la mayoría de las veces son solamente hipótesis basadas en modelos de inferencia utilizando subrogados de la biodiversidad. Esto se debe a que no se conoce la identidad precisa de las especies y, por otro lado, a que la mayoría de las veces tampoco se sabe con precisión dónde se encuentran. Richardson y Whittaker (2010) indican que la primera es una deficiencia del conocimiento taxonómico (Déficit Linneano), y la otra una deficiencia del conocimiento de su distribución geográfica (Déficit Wallaceano).

Los ejemplos mostrados en las líneas anteriores son utilizados para mostrar aspectos contemporáneos que tienen que ver, tanto con la investigación científica como con la problemática conocida como la crisis de la biodiversidad. Por un lado, existe una acelerada pérdida de especies, especialmente causada por las actividades humanas o por la alteración y reducción de su hábitat natural, que las hace 
más susceptibles a enfermedades o depredadores. Por otro lado, está el profundo desconocimiento que se tiene de gran parte de ellas, solamente conocidas por los datos de su descripción original.

A pesar de la urgencia por conocer la biodiversidad faltante, el trabajo que se realiza para recolectar, reconocer y describir a las especies es poco entendido y con frecuencia menospreciado. En diversas instituciones académicas y gubernamentales relacionadas con el conocimiento y manejo de la biodiversidad, el trabajo de los taxónomos no es considerado como una actividad científica, sino una técnica o un arte, aunque después utilicen los nombres científicos propuestos por ellos. Los nombres científicos constituyen, en esencia, la hipótesis de trabajo del taxónomo (Ertter, 2000), misma que es utilizada para desarrollar una amplia gama de líneas de investigación; por ejemplo, biología molecular, conservación, ecología o evolución.

Este trabajo presenta un escenario del papel de la taxonomía vegetal en algunos de los aspectos actualmente discutidos sobre la problemática de la biodiversidad. La hipótesis que se sigue en este trabajo, es que evitando la crisis que enfrenta actualmente la taxonomía, se minimizará de manera importante la crisis de la biodiversidad. Lo anterior, debido a que conociendo mejor la diversidad vegetal y su distribución geográfica, se pueden proponer mejores estrategias para su conservación por medio de una red más eficiente de áreas naturales protegidas y aspectos relacionados con su manejo. Se pondrá énfasis en aquellos aspectos donde se considera que la taxonomía juega un papel preponderante, y se presentarán estrategias actualizadas que la han revitalizado y ubicado como una de las grandes esperanzas contra esta crisis de la biodiversidad. Las ideas están organizadas en tres secciones, que describen: (1) el papel que han jugado los taxónomos mexicanos en el conocimiento de la flora de México y cuál es el capital humano con que se cuenta en la actualidad, (2) el conocimiento que se tiene actualmente sobre la flora de México y su relevancia en las estrategias de conservación de la biodiversidad y, (3) líneas de investigación novedosas que podrían complementar el conocimiento taxonómico para definir las zonas más importantes de riqueza y endemicidad (hotspots) que perfeccionen o actualicen nuestro Sistema Nacional de Áreas Naturales Protegidas.

\section{El papel del taxónomo en la ciencia y la sociedad}

Diversos trabajos han puesto énfasis en explicar la importancia del trabajo de un taxónomo (Disney, 1983; Maxted, 1992; Watson, 1997). El trabajo fundamental del taxónomo vegetal se centra en describir la diversidad y ordenarla en un sistema de clasificación que sirva como referencia para otras ramas de la biología. Otro objetivo que encara el taxónomo es utilizar dicha clasificación para generar herramientas para identificar la diversidad de plantas y, de esa manera, ayudar a otras personas en su identificación.
Existen diversas disciplinas en biología que dependen de una correcta identificación taxonómica de los organismos que estudian. Por ejemplo, cuando se trata de explicar posibles patrones de evolución, se requiere saber claramente con que especie se está trabajando y cuáles son sus parientes más cercanos. En biología molecular, la secuenciación genética de varias especies exige que hayan sido identificadas correctamente. En química, el estudio de productos naturales específicos (p. ej. alcaloides o sesquiterpenos) requiere previamente saber las especies que los contienen y saber identificarlas en el campo para obtener el material adecuado para su extracción. Estos investigadores obtienen la información de sus especies con el estudio de los taxónomos que trabajan en un herbario y de sus publicaciones.

Es importante resaltar que el trabajo realizado por los taxónomos no consiste solamente en describir nuevas especies, sino en gran medida, en ordenar y clasificar correctamente numerosas especies descritas previamente. Cualquiera que ha intentado hacer una revisión taxonómica, se ha enfrentado a las dificultades de desentrañar todo lo escrito por los estudiosos de la biodiversidad de los siglos XVIII, XIX y XX, e inclusive antes; no es tarea fácil tratar de interpretar descripciones o ejemplares de herbario no siempre completos para tomar conclusiones apropiadas, lo que consume una gran cantidad de tiempo y esfuerzo. Finalmente, está el costo del trabajo de campo, necesario para entender la compleja naturaleza de las especies; que muy pocas veces es adecuadamente valorado.

El problema de la desaparición de los taxónomos en el mundo, y México no es la excepción, se debe en gran parte a los mecanismos de evaluación de la producción académica, a los ingresos obtenidos mediante recursos extraordinarios (subsidios a proyectos) y al factor de impacto de las revistas donde publican los resultados de su investigación. Un científico será mejor valorado mientras más publicaciones genere, más recursos consiga para sus investigaciones e incremente su tasa de publicación en revistas de alto impacto internacional. Por ejemplo, publicar en la actualidad un inventario florístico es considerado como algo que merma su calidad académica, inclusive para varias revistas que anteriormente los aceptaban, aunque nadie pone en duda lo valioso que es cuantificar el número y diversidad de especies de un sitio. Tampoco se toma en consideración todo el tiempo que fue necesario para realizar dicho inventario, del cual probablemente se descubrieron una o más especies nuevas para la ciencia. El carácter local de la investigación tampoco da elementos para ser considerado de impacto internacional y ser evaluado con los estándares modernos, aunque su información eventualmente sea incorporada a los grandes y complejos bancos de información consultados en distintas regiones del mundo, gracias al ciberespacio (GBIF, REMIB, Tropicos, Unibio, etc.). Desafortunadamente, el taxónomo es considerado en la actualidad un académico devaluado, tanto económicamente (no consigue recursos importan- 


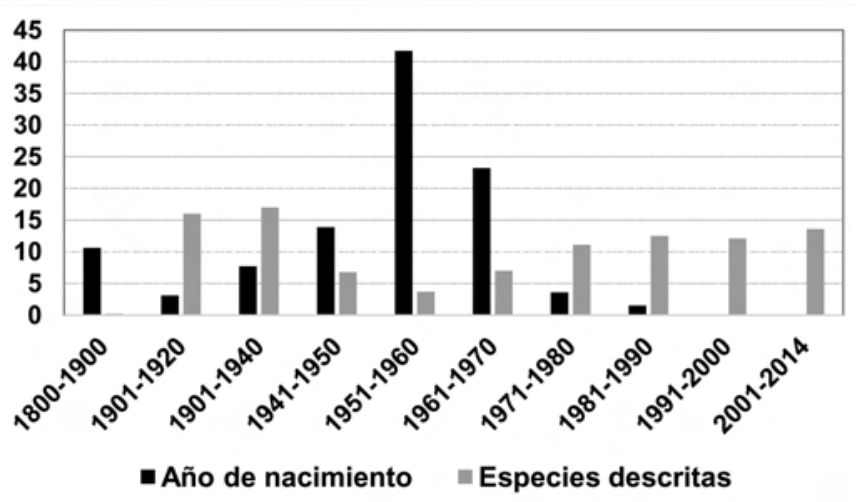

Figura 2. Año de nacimiento de los taxónomos mexicanos que han descrito especies nuevas para la ciencia $(N=280$, barras negras) y número de especies de plantas vasculares nuevas para la ciencia descritas en el mismo periodo (barras grises). Los valores están dados en porcentajes.

tes), como científicamente (no publica en revistas de alto impacto internacional y, por lo tanto, carece de citas a sus publicaciones). Debido a esta concepción, los taxónomos difícilmente pueden conseguir apoyo económico para tareas tan importantes como son los inventarios florísticos, aunque algunas de las especies descritas por ellos sean citadas cientos de veces en revistas de alto impacto, pero sin mencionar la fuente original donde fueron descritas. Actualmente, la llamada crisis de la biodiversidad puede ser considerada también la de la taxonomía, pues los taxónomos, quienes son los responsables de generar el conocimiento básico para ayudar a evitar esta crisis de la biodiversidad, son tan escasos que podrían ser considerados también una "especie en peligro de extinción".

Parte de la evidencia de la reducción en el número de taxónomos involucrados en la descripción de la diversidad vegetal en México, se puede encontrar al analizar el número de botánicos que se han involucrado en la descripción de especies nuevas para la ciencia (Figura 2). Un recuento realizado por Villaseñor et al. (2008), señala que el número de taxónomos involucrados en el conocimiento y descripción de la riqueza florística de México asciende a 3,315 y solamente 280 (8.4\%) son mexicanos. El número de taxónomos mexicanos involucrados en la descripción de especies nuevas se ha reducido drásticamente en las últimas décadas (Figura 2). De hecho, el porcentaje de taxónomos mexicanos jóvenes en la actualidad es más o menos el mismo que se tenía a principios del siglo pasado, cuando la actividad científica en México era incipiente. Se puede deducir que gran parte de la descripción de la diversidad vegetal en el país la están llevando a cabo taxónomos mexicanos con edades promedio entre los 40 y 70 años, y al parecer, la formación de nuevos especialistas está disminuyendo de manera considerable (Figura 2). Otra posible explicación puede ser que la actividad de descripción de nuevos taxones no es una tarea sencilla y que se requiere de un largo proceso de madurez intelectual y especialización, para poder describir de manera eficiente esta biodiversidad.

Importancia del trabajo de herbario. En un estudio realizado en 2010 (Bebber et al., 2010), se encontró que de una muestra de especies nuevas publicadas entre 1970 y 2010, solamente $16 \%$ de ellas se basaron en material recientemente recolectado, mientras que el $84 \%$ restante fue descrito con material depositado en los herbarios, recolectado cinco o más décadas antes. Las conclusiones de dicho estudio son, que gran parte de la biodiversidad vegetal aún no descrita puede ya estar almacenada en los herbarios, por lo que se debe poner énfasis en el estudio y curación del material ya existente en dichas colecciones, sin descuidar por supuesto, la exploración de áreas consideradas prioritarias. ¿Pero quiénes son las personas entrenadas y con la suficiente experiencia para estudiar adecuadamente las colecciones científicas y ubicar las especies no descritas? Este papel no puede ser desempeñado por otros investigadores más que por los taxónomos, quienes a su vez tienen experiencia para descubrir en el campo las plantas que representan la rareza de una flora y donde seguramente se esconden las novedades todavía no descritas de la biodiversidad, así como ubicar las regiones donde estas plantas se encuentran.

Repitiendo de alguna forma parte del análisis realizado por Bebber et al. (2010) con las especies descritas como nuevas para la ciencia en el último lustro en México (20092014), se pueden discutir varios aspectos (Cuadro 2). En primer lugar, al considerar aquellas especies que fueron recolectadas prácticamente el mismo año en que fueron descritas como nuevas especies, pocas de ellas se publicaron durante los primeros dos años. Lo que es importante hacer notar, como señalan Bebber et al. (2010), es que muy probablemente mucha de la biodiversidad aún desconocida se encuentra ya depositada en las colecciones, pero su rescate y descripción no es tarea sencilla sino altamente especializada. Prácticamente, la descripción de la mitad de la diversidad de

Cuadro 2. Periodo de tiempo entre la recolecta de material que representa una especie nueva para la ciencia, su incorporación en el herbario y la publicación respectiva. El periodo analizado comprende los años 2009 a $2014(N=284)$.

\begin{tabular}{ccc}
\hline $\begin{array}{c}\text { Tiempo entre recolecta } \\
\text { y publicación (años) }\end{array}$ & $\begin{array}{c}\text { Número } \\
\text { de especies }\end{array}$ & Porcentaje \\
\hline 1 & 26 & 9.1 \\
2 & 43 & 15.1 \\
$3-10$ & 77 & 27.1 \\
$11-20$ & 34 & 12.0 \\
$21-30$ & 47 & 16.5 \\
$31-40$ & 11 & 3.9 \\
$41-50$ & 7 & 2.5 \\
51 o más & 11 & 3.9 \\
\hline
\end{tabular}


especies de plantas con flores en el último lustro, se basó en material recolectado 11 o hasta más de 100 años atrás.

No debe olvidarse que los herbarios, además de registrar la diversidad vegetal desde un punto de vista taxonómico, albergan información adicional de utilidad para muchas otras áreas de investigación. Ya desde mediados del siglo pasado se destacaba el potencial de información que las colecciones tenían para la búsqueda; por ejemplo, de especies comestibles que se adicionarán a la dieta (von Reis Altschul, 1977) o que proporcionarán drogas útiles para incrementar el perfil de medicamentos utilizados. Pero el banco de información que constituyen tales colecciones solamente es útil si está bien curado; es decir, si los especímenes han sido revisados y correctamente identificados por los taxónomos especialistas. La falta de una buena curación de la información taxonómica puede generar errores, como los que ejemplifica Bortolus (2008), para el caso del uso de una taxonomía errónea en ecología.

\section{El conocimiento de la diversidad vegetal y su impor- tancia en la conservación}

Algunos conceptos, tanto modernos como otros ya discutidos desde hace muchos años, permiten evaluar patrones de diversidad florística en México y destacar su relevancia en el papel que juegan como escenario de especiación y de la conservación de la biodiversidad. Aquí se discuten a manera de ejemplo dos de ellos.

Hotspots. Un hotspot, como fue inicialmente concebido, caracteriza una región que cuenta con una extraordinaria biodiversidad restringida a su territorio (endémica), sujeta a elevados niveles de transformación de su hábitat natural (Myers, 1988). Más recientemente, Myers et al. (2000) redefinieron el concepto al definirlo como aquella región con al menos 1,500 especies de plantas endémicas que sobreviven en $30 \%$ o menos de su hábitat natural, debido a la transformación del ambiente por las actividades humanas. Los estudios más recientes han revelado la existencia de 35 hotspots de biodiversidad (Mittermeier et al., 2011). El continente americano contiene ocho de esos hotspots, cuatro de ellos ubicados en Sudamérica (la región tropical de Los Andes, la región Tumbes-Choco-Magdalena en Colombia, el Cerrado de Brasil y los bosques templados de clima mediterráneo de Chile); otro hotspot lo constituye el archipiélago del Caribe. En Norteamérica se identifican dos hotspots; uno de ellos es la Provincia Florística de California (que incluye una pequeña región en el extremo noroccidental del territorio nacional), característica por su clima mediterráneo, y el otro son las montañas del suroeste de los Estados Unidos que se continúan hasta el norte de México, conocido en su conjunto como los bosques templados de la región Madrense. El octavo hotspot lo constituye la región Mesoamericana, que abarca desde el sureste de México hasta Centroamérica.
Los tres hotspots identificados en América y que abarcan territorio de México difieren substancialmente en su riqueza florística (Mittermeier et al., 2011). La Provincia Florística de California tiene un nivel de endemismo de alrededor de $57.1 \%$, tomando como base un estimado de 3,500 especies. Por su parte, los bosques templados de la Región Madrense contienen un número estimado en más de 5,000 especies, de las cuales casi $80 \%$ son endémicas. Finalmente, la Región Mesoamericana debe albergar alrededor de 17,000 especies, con un nivel de endemismo cercano al $18 \%$.

México registra en la actualidad 21,841 especies de plantas con flores, de las cuales $11,001(50.4 \%)$ son endémicas de su territorio (Villaseñor y Ortiz, 2014). Con base en estas cifras, se le podría considerar como un hotspot, ya que supera los valores de endemicidad de varios de los hotspots considerados por Mittermeier et al. (2011), además de que la superficie de vegetación que ha sido transformada alcanza valores cercanos al 30\%. Evaluando la carta de uso del suelo y vegetación del 2005, la superficie sin vegetación natural de acuerdo con el INEGI (2005) alcanza 28\% del territorio nacional. A una escala más fina, siete estados de los 32, en que políticamente está dividido México, registran en su territorio casi 1,500 o más especies endémicas de México, valores que son comparables a los considerados para categorizar las zonas como hotspots, prácticamente todos ellos con más del $30 \%$ de su superficie transformada y con la vegetación natural ya perdida: Chiapas (1,486 especies endémicas, $45 \%$ de su territorio transformado), Durango (1,456, 13\%), Guerrero (2,296, 33\%), Jalisco (2,794, 37\%), Michoacán (2,223, 39\%), Oaxaca $(3,672,33 \%)$ y Veracruz $(1,870,80 \%)$. Ante este escenario cabe preguntar: ¿qué porcentaje de la diversidad total y del endemismo, tanto a nivel nacional como estatal persiste en las áreas todavía con vegetación natural? Los inventarios florísticos, llevados a cabo por los taxónomos, podrían revelar la diversidad y cantidad de taxa que aún existen en tales remanentes de vegetación natural. Desafortunadamente, cada vez se observa un menor interés institucional por apoyar estas actividades de inventario, contrario a lo que se observaba, por ejemplo, en la década de los 80 .

Biomas. Los inventarios florísticos brindan información del número de especies vegetales observadas en una región. Cuando se combina la diversidad florística y sus atributos (forma de crecimiento, fidelidad a una determinada asociación vegetal, etc.), con factores como el clima y el sustrato, se generan patrones complejos a gran escala conocidos como formaciones vegetales o biomas (Krebs, 1978; Gurevitch et al., 2002). Los biomas son grandes regiones biogeográficas que difieren de otras en la estructura de su vegetación y en sus especies vegetales dominantes. El término de bioma fue acuñado por el botánico y ecólogo Frederic Edward Clements (1916) y su concepto ha demostrado ser de gran importancia en los estudios de biodiversidad. Por 
ejemplo, la clasificación en biomas ha permitido determinar el número de especies vegetales distintas en un lugar con clima o sustrato geológico específico, así como las formas de vida que registran sus elementos dominantes (Villaseñor y Ortiz, 2014).

Los biomas están conformados por asociaciones vegetales con historias evolutivas particulares, que pueden contener un número importante de linajes antiguos (conocidos como "museos") o estar mejor representados por radiaciones evolutivas relativamente recientes (conocidos como "cunas"), caracterizados por activos procesos de evolución (p.ej., Magallón et al., 2014). Conocer la diversidad florística de los biomas es un paso importante en la comprensión de su historia evolutiva; ambos aspectos permitirían tomar mejores decisiones para su conservación o, más recientemente, evaluar los efectos del cambio climático en su composición florística y capacidad de resiliencia.

No hay un consenso global en el número de biomas existentes en el mundo y su número se estima entre 14 y 19 (Krebs, 1978; Olson et al., 2001; Gurevitch et al., 2002; Pennington et al., 2004). A nivel nacional, Challenger y Soberón (2008) discuten la presencia de siete tipos principales de vegetación o categorías que podrían homologarse con el concepto de bioma, mientras que Villaseñor y Ortiz (2014) mencionan la existencia de cinco biomas. El cuadro 3 propone una homología entre estas propuestas y como puede observarse, en México existen casi todos los tipos de biomas identificados en el mundo, lo que sin lugar a dudas está relacionado con la evolución y diversificación de la megadiversidad encontrada en su territorio (Rzedowski, 1991; Sarukhán y Dirzo, 1992).

Los biomas no han sido considerados por los taxónomos y para muy pocos de ellos se han indicado sus componentes florísticos (p.ej., Villaseñor et al., 2003, Ibarra-Manríquez et al., aceptado). Muchos biomas se han caracterizado solamente por sus rasgos ecológicos; sin embargo, en la actualidad la riqueza florística de México se conoce mejor que hace unas décadas, cuando fueron publicados los trabajos más importantes sobre tipos de vegetación (p.ej., Miranda y Hernández-X., 1963; Gómez-Pompa, 1965; Pennington y Sarukhán, 1968; Rzedowski, 1978). Desafortunadamente, la problemática de encontrar el consenso entre los tipos de vegetación discutidos por dichos trabajos ha dificultado la evaluación de su diversidad florística. Por el contrario, el uso de los biomas como unidad de estudio ha permitido evaluar con más precisión cómo se distribuye la riqueza florística entre ellos (Villaseñor y Ortiz, 2014).

Las cifras de riqueza y endemicidad presentadas por Villaseñor y Ortiz (2014) para cada uno de los cinco biomas considerados en el país, revelan que todos ellos destacarían como hotspots de diversidad y endemicidad. Por otra parte, solamente de manera regional se ha documentado el impacto que dichos biomas y sus comunidades vegetales están sufriendo por las actividades humanas. Pueden servir como ejemplos el estado de amenaza que sufre el bioma húmedo de montaña (referido como bosque mesófilo de montaña), discutido por Sánchez-Ramos y Dirzo (2014), o la tasa de deforestación que Dirzo y García (1992) documentaron para la región de Los Tuxtlas, en el estado de Veracruz, donde predominaba el bioma tropical húmedo (selva tropical húmeda).

Aunque las cifras publicadas revelan altos valores de diversidad florística entre los biomas (Villaseñor y Ortiz,

Cuadro 3. Biomas reconocidos a nivel mundial y su correspondencia con los cinco biomas reconocidos por Villaseñor y Ortiz (2014), o los siete principales tipos de vegetación discutidos por Challenger y Soberón (2008) en México.

\begin{tabular}{|c|c|c|}
\hline A nivel mundial & $\begin{array}{l}\text { Challenger y } \\
\text { Soberón (2008) }\end{array}$ & $\begin{array}{l}\text { Villaseñor y } \\
\text { Ortiz (2014) }\end{array}$ \\
\hline Tundra & - & - \\
\hline Bosques boreales-taigas & - & - \\
\hline $\begin{array}{l}\text { Bosques templados } \\
\text { de coníferas }\end{array}$ & $\begin{array}{l}\text { bosques templados de } \\
\text { coníferas y latifoliadas }\end{array}$ & $\begin{array}{l}\text { bosques } \\
\text { templados }\end{array}$ \\
\hline $\begin{array}{l}\text { Bosques templados de } \\
\text { hoja ancha y } \\
\text { bosques mixtos }\end{array}$ & $\begin{array}{l}\text { bosques templados de } \\
\text { coníferas y latifoliadas }\end{array}$ & $\begin{array}{l}\text { bosques } \\
\text { templados }\end{array}$ \\
\hline $\begin{array}{l}\text { Pastizales templados, } \\
\text { sabanas y matorrales }\end{array}$ & pastizales & $\begin{array}{l}\text { bosques } \\
\text { templados } \\
\text { (en parte) }\end{array}$ \\
\hline $\begin{array}{l}\text { Bosques y matorrales } \\
\text { mediterráneos }\end{array}$ & - & $\begin{array}{l}\text { matorrales } \\
\text { xerófilos }\end{array}$ \\
\hline $\begin{array}{l}\text { Bosques tropicales y } \\
\text { subtropicales de coníferas }\end{array}$ & - & $\begin{array}{l}\text { bosques } \\
\text { templados }\end{array}$ \\
\hline $\begin{array}{l}\text { Bosques tropicales y } \\
\text { subtropicales de } \\
\text { hoja ancha }\end{array}$ & $\begin{array}{l}\text { bosques tropicales } \\
\text { perennifolios }\end{array}$ & $\begin{array}{l}\text { bosques } \\
\text { tropicales } \\
\text { húmedos }\end{array}$ \\
\hline $\begin{array}{l}\text { Bosques tropicales y } \\
\text { subtropicales secos }\end{array}$ & $\begin{array}{l}\text { bosques tropicales } \\
\text { caducifolios }\end{array}$ & $\begin{array}{l}\text { bosques } \\
\text { tropicales } \\
\text { estacionalmente } \\
\text { secos }\end{array}$ \\
\hline $\begin{array}{l}\text { Pastizales, sabanas y } \\
\text { matorrales tropicales y } \\
\text { subtropicales }\end{array}$ & pastizales & $\begin{array}{l}\text { bosques } \\
\text { tropicales } \\
\text { húmedos } \\
\text { (en parte) }\end{array}$ \\
\hline Matorrales desérticos & matorrales xerófilos & $\begin{array}{l}\text { matorrales } \\
\text { xerófilos }\end{array}$ \\
\hline Manglares & humedales & $\begin{array}{l}\text { bosques } \\
\text { tropicales } \\
\text { húmedos }\end{array}$ \\
\hline $\begin{array}{l}\text { Pastizales y sabanas } \\
\text { inundables }\end{array}$ & pastizales & $\begin{array}{l}\text { bosques } \\
\text { tropicales } \\
\text { húmedos } \\
\text { (en parte) }\end{array}$ \\
\hline $\begin{array}{l}\text { Pastizales y matorrales } \\
\text { de grandes altitudes }\end{array}$ & pastizales & $\begin{array}{l}\text { bosques } \\
\text { templados }\end{array}$ \\
\hline $\begin{array}{l}\text { Bosque de neblina o } \\
\text { bosque nublado }\end{array}$ & $\begin{array}{l}\text { bosque mesófilo } \\
\text { de montaña }\end{array}$ & $\begin{array}{l}\text { bosque húmedo } \\
\text { de montaña }\end{array}$ \\
\hline
\end{tabular}



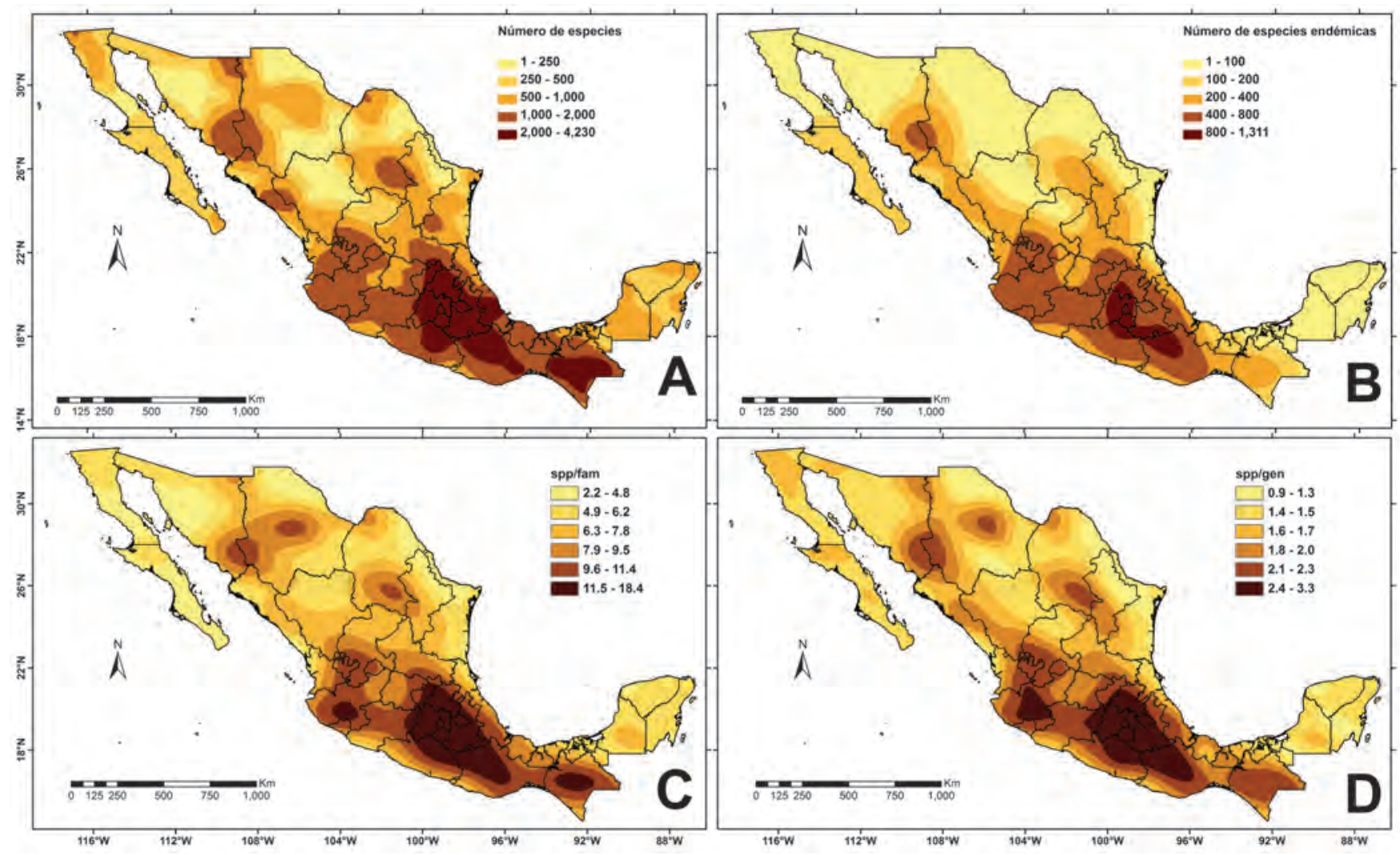

Figura 3. A) Patrones de riqueza florística total, B) de riqueza de endemismos, C) proporción de la riqueza total de especies/familias y D) relación de la riqueza total de especies/géneros. Los mapas fueron elaborados con la técnica de interpolación Kriging, con base en la riqueza conocida, en cuadros de $1^{\circ}$ de latitud y longitud.

2014), poco se sabe sobre su distribución geográfica a lo largo de sus territorio. Solamente con más exploración de campo y la síntesis de dicho trabajo en los inventarios florísticos se podrá, en un futuro, conocer con mayor precisión cómo se distribuye la riqueza y endemismo a lo largo de la superficie ocupada por cada bioma. Pero será necesaria una mayor labor de identificación del material recolectado, lo que a su vez demanda la experiencia de los taxónomos. Se ha estimado que un taxónomo trabajando en actividades de inventario durante varios años en una pequeña región, puede reconocer entre 200 y 500 especies; las otras especies se conocerán solamente mediante la labor de identificación botánica, mucha de ella apoyada mediante el cotejo con material de herbario previamente identificado (Villaseñor y Murguía-Romero, 1992).

\section{Nuevos enfoques en el estudio de la biodiversidad}

La información sobre la biodiversidad, sobre todo la registrada en las colecciones científicas, es cada vez más demandada por especialistas de diferentes áreas del conocimiento. Ello ha obligado a buscar mecanismos para integrar dicha información de la mejor manera posible, sintetizarla y hacerla accesible para todos los interesados. La búsqueda efi- ciente de tales mecanismos ha generado lo que se conoce en la actualidad como la informática de la biodiversidad (Canhos et al., 2004; Soberón y Peterson, 2004). La captura de la información biológica almacenada en las colecciones es la primera etapa para hacerla accesible y útil. El desarrollo y uso de las computadoras en esta empresa ha sido crucial, y desde los intentos pioneros en Australia en la década de los 70, ahora es casi una práctica común que la mayoría de las colecciones científicas estén involucradas en la generación de bases de datos electrónicas con la información de sus acervos (p.ej., Tropicos, UNIBIO). Inclusive, esta iniciativa se ha llevado hasta las esferas sociales o gubernamentales, creándose instituciones como ERIN en Australia, INBIO en Costa Rica o CONABIO en México, que administran acervos importantes de información acerca de la biodiversidad de sus países. Un paso adelante es el esfuerzo llevado a cabo por algunas de esas instituciones por conjugar la información de muchas bases de datos administradas por las colecciones científicas, de tal manera que el intercambio de información se vuelva eficiente y accesible para usuarios potenciales. Como ejemplo están la REMIB de la CONABIO, que permite acceder a información de un gran número de colecciones, tanto botánicas como zoológicas, principalmente mexicanas, mediante una sola consulta, 
o GBIF con aplicaciones similares a la REMIB, pero en un plano mundial. Las bases de datos generadas con la información de los especímenes de las colecciones han sido de gran utilidad para el desarrollo de nuevas líneas emergentes de investigación, como son los análisis de patrones de diversidad y los modelos generados para estimarla (p.ej., CruzCárdenas et al., 2013; Figura 3), o los estudios para evaluar la distribución potencial o el nicho ecológico de las especies (Franklin, 2009; Peterson et al., 2011).

La información primaria de las bases de datos que genera la informática de la biodiversidad proviene de la información taxonómica (nombres de las especies y su clasificación en un sistema jerárquico) y lo adicional que contienen los especímenes resguardados en las colecciones (por ejemplo fecha, hábitat y localidad de recolecta). Es claro entonces, que esta nueva línea de investigación difícilmente pudo haber surgido sin las colecciones científicas y su información contenida en los especímenes que albergan. En consecuencia, el éxito de la informática de la biodiversidad depende de qué tan consistente y confiable es la información taxonómica en las colecciones; pero ya se mencionó que la curación de un herbario, es decir, el mantenimiento actualizado de los especímenes, tanto en su calidad de almacenamiento (cuidados técnicos) como de información taxonómica (correcta identificación), depende en gran medida de los taxónomos asociados a dichas colecciones. Lamentablemente, en muchos casos, la información que se incorpora a las bases de datos es de dudosa confianza para ser usada en los análisis de la biodiversidad. Indudablemente, esta nueva línea de investigación no avanza al ritmo esperado cuando se enfrenta a los problemas de capturar la información sin el adecuado nivel de curación que le brindan los expertos. El cuadro 4

Cuadro 4. Nivel de curación (adaptado de McGinley, 1993) de una colección (herbario) con base en el estado de curación de sus especímenes.

\begin{tabular}{cl}
\hline Nivel & \multicolumn{1}{c}{ Estado de curación } \\
\hline 1 & $\begin{array}{l}\text { Especímenes en mal estado, la mayoría sin montar y sin } \\
\text { identificar. La colección necesita atención inmediata. }\end{array}$ \\
2 & $\begin{array}{l}\text { Especímenes sin montar pero muchos ya identificados; } \\
\text { para efectos prácticos inaccesibles para los especialistas. }\end{array}$ \\
3 & $\begin{array}{l}\text { Especímenes montados pero sin identificar, la mayoría } \\
\text { todavía inaccesibles para los especialistas. }\end{array}$ \\
4 & $\begin{array}{l}\text { Especímenes montados, identificados a familia o género, } \\
\text { accesibles para los especialistas. }\end{array}$ \\
5 & $\begin{array}{l}\text { Especímenes identificados y montados, pero se necesita } \\
\text { actualización taxonómica y nomenclatural. }\end{array}$ \\
6 & $\begin{array}{l}\text { Especímenes bien curados e identificados de acuerdo con } \\
\text { estándares nacionales o internacionales. }\end{array}$ \\
7 & Especímenes de nivel 6 incorporados a una base de datos. \\
& $\begin{array}{l}\text { Especímenes del nivel } 7 \text { con las localidades } \\
\text { georreferenciadas. }\end{array}$ \\
\hline
\end{tabular}

Cuadro 5. Datos reportados en internet por GBIF (GBIF.org) a partir de bases de datos generadas en la colección del Herbario Nacional (MEXU).

\begin{tabular}{lccc}
\hline $\begin{array}{l}\text { Colección o base } \\
\text { de datos }\end{array}$ & $\begin{array}{c}\text { Total de } \\
\text { registros }\end{array}$ & $\begin{array}{c}\text { Registros } \\
\text { georreferenciados }\end{array}$ & $\begin{array}{c}\text { Registros en } \\
\text { Nivel 8 de } \\
\text { curación (\%) }\end{array}$ \\
\hline Briofitas & 40,472 & 29,760 & 73.5 \\
Plantas acuáticas & 35,489 & 28,010 & 78.9 \\
Plantas vasculares & 31,082 & 9,402 & 30.2 \\
Asteraceae & 30,985 & 30,889 & 99.7 \\
Leguminosae & 11,955 & 3,440 & 28.8 \\
Asclepiadaceae & 7,127 & 7,122 & 99.9 \\
\hline
\end{tabular}

presenta una clasificación (adaptada de McGinley, 1993), de los herbarios de acuerdo con el nivel de curación de sus especímenes. Es evidente que mientras mejor curada está una colección, más importante será su participación en estas nuevas líneas emergentes.

Un ejemplo de la evaluación que se podría hacer de las colecciones para considerar su potencial de participación en estudios no de naturaleza estrictamente taxonómica o florística, resulta de la consulta de uno de los buscadores de bases de datos más utilizado en la actualidad (GBIF.org, consultada el 18 de septiembre de 2014). Se obtuvo la información reportada para las seis bases de datos con mayor número de registros provenientes del Herbario Nacional (MEXU) del Instituto de Biología de la UNAM (Cuadro 5). Dichos bancos de información podrían considerarse en los niveles 7 u 8 del cuadro 4, pues se esperaría que la información esté bien curada (nivel 6) y almacenada en bases de datos (nivel 7).

Como se observa en el cuadro 5, las colecciones específicas con mayor número de registros curados en el herbario MEXU presentan diferencias en sus estándares, con respecto a los últimos niveles indicados en el cuadro 4. Hay colecciones (Asclepiadaceae s.s. y Asteraceae) que calificarían como de nivel 8 (bien curadas, identificadas y georreferenciadas), mientras que otras presentan niveles intermedios entre los niveles 7 y 8 (inclusive más bajos). Sería interesante repetir este ejercicio con todas las colecciones nacionales que albergan la información en torno a la riqueza florística de México. Indudablemente dicho ejercicio reflejaría el gran esfuerzo que están haciendo los taxónomos por documentarla y ponerla al servicio de la sociedad. Solamente así se podría evaluar mejor su contribución y el uso que se está haciendo de ella. Por ejemplo, son interesantes las cifras reportadas por Suarez y Tsutsui (2004), de artículos publicados en revistas de muy alto nivel internacional que basaron sus estudios en material almacenado y curado por los taxónomos. Desafortunadamente, en la mayoría de los trabajos revisados por estos autores (y una revisión personal de muchos en el plano nacional), difícilmente citan la participación del especialista en la identificación del material utilizado. 


\section{Reflexiones finales}

Muchas de las ideas y ejemplos expuestos a lo largo del texto enfatizan el papel preponderante que los taxónomos juegan en el conocimiento básico de la biodiversidad y en su potencial contribución para mitigar su crisis. Es evidente que otras ramas de la biología dependen del trabajo curatorial en las colecciones científicas, pero el escaso reconocimiento que existe en fechas recientes por dicha actividad no motiva a jóvenes estudiantes hacia el área de la taxonomía. Una manera de hacerlo es resaltando el planteamiento de las hipótesis que solamente la actividad realizada por los taxónomos ayudaría a evaluar: con base en los mapas de la figura 3, podría postularse que la diversidad faltante para completar el inventario florístico del país debería buscarse en las áreas todavía con poca exploración y con bajo número de especies registradas. Sin embargo, siguiendo la idea de que la diversidad se auto promueve, podría igualmente postularse que las áreas donde está ocurriendo una alta tasa de especiación (representada por el cociente especies/familia o especies/género; Figura 3C, D), es donde gran parte de las especies aún desconocidas deberían buscarse. Solamente con más exploración de campo, identificación y, en su caso, descripción de taxa nuevos para la ciencia, podrá contestarse cuál de las dos hipótesis es la correcta. Pero con la escasez de taxónomos difícilmente se podrán evaluar, y lo más probable es que la destrucción acelerada de los ambientes naturales impida aproximarse a cifras más confiables de la biodiversidad existente en nuestro país.

Es importante resaltar que otras áreas de investigación, como Biogeografía, Ecología, Evolución, Informática de la Biodiversidad, Modelos de Nicho Ecológico o Química, indudablemente sufrirán una crisis parecida a la de la biodiversidad, cuando la información que requieran para sus estudios adolezca del suficiente grado de confianza que el trabajo de los taxónomos les proporciona. En consecuencia, indudablemente la crisis de la biodiversidad, como la concebimos en la actualidad, va paralela con la crisis de la taxonomía, y tristemente, no se ven intenciones de revertir ni una ni la otra. Es imperativo, en consecuencia, enfatizar la relevancia de que los datos taxonómicos confiables son fundamentales para todo lo relacionado con la investigación, uso y conservación de la diversidad vegetal. La motivación a jóvenes interesados en revertir estas tendencias, mediante su formación como futuros taxónomos será una de las mejores estrategias para cumplir el compromiso de contar con un mejor conocimiento para la conservación de nuestro gran legado natural.

\section{Agradecimientos}

Las ideas plasmadas en el texto fueron generadas a partir de una serie de conferencias sobre el papel de la taxonomía en el conocimiento de la biodiversidad. Agradezco la lectura crítica y la motivación de colegas como Francisco Espinosa García y Guillermo Ibarra Manríquez (Centro de Investigaciones en Ecosistemas, UNAM), y Teresa Terrazas (Instituto de Biología, UNAM), que lograron concretar la discusión generada en las pláticas en este manuscrito. Como siempre, la asistencia técnica del Biólogo Enrique Ortiz Bermúdez fue crucial para culminar este ensayo, sobre todo en el manejo de la información y en la elaboración de los mapas. Los comentarios de Joselin Cadena, Verónica Juárez y Victoria Sosa enriquecieron substancialmente el manuscrito.

\section{Literatura citada}

Bebber D.P., Carine M.A., Wood J.R.I., Wortley A.H., Harris D.J., Prance G.T., Davidse G., Paige J., Pennington T.D., Robson N.K.B. y Scotland R.W. 2010. Herbaria are a major frontier for species discovery. Proceedings of the National Academy of Sciences of the United States of America 107:22169-22171.

Bortolus A. 2008. Error cascades in the biological sciences: the unwanted consequences of using bad taxonomy in ecology. AMBIO 37:114-118.

Canhos V.P., Souza S., Giovanni R. y Canhos D.A.L. 2004. Global biodiversity informatics: setting the scene for a "new world" of ecological modeling. Biodiversity Informatics 1:1-13.

Challenger A. y Soberón J. 2008. Los ecosistemas terrestres. En: Comisión Nacional para el Conocimiento y Uso de la Biodiversidad. Capital Natural de México. Vol. I: Conocimiento Actual de la Biodiversidad, pp. 87-108, Comisión Nacional para el Conocimiento y Uso de la Biodiversidad, México, D.F.

Clements F.E. 1916. Plant succession: an analysis of the development of vegetation. Carnegie Institution of Washington Publication 242:1-512

CONABIO. Comisión Nacional para el Conocimiento y Uso de la Biodiversidad. 2012. Estrategia Mexicana para la Conservación Vegetal, 2012-2030. Comisión Nacional para el Conocimiento y Uso de la Biodiversidad, México, D.F.

CONABIO, CONANP, SEMARNAT. Comisión Nacional para el Conocimiento y Uso de la Biodiversidad, Comisión Nacional de Áreas Nacionales Protegidas, Secretaría de Medio Ambiente y Recursos Naturales. 2008. Estrategia Mexicana para la Conservación Vegetal: Objetivos y Metas. Comisión Nacional para el Conocimiento y Uso de la Biodiversidad. México, D.F.

Cruz-Cárdenas G., Villaseñor J.L., López-Mata L. y Ortiz E. 2013. Distribución espacial de la riqueza de especies de plantas vasculares en México. Revista Mexicana de Biodiversidad 84:1189-1199.

Dirzo R. y García M.C. 1992. Rates of deforestation in Los Tuxtlas, a Neotropical area in Southeast Mexico. Conservation Bio$\log y$ 6:84-90.

Disney R.H.L. 1983. A synopsis of the taxonomist's tasks, with particular attention to phylogenetic cladism. Field Studies 5:841-865.

Ertter B. 2000. Floristic surprises in North America North of Mexico. Annals of the Missouri Botanical Garden 87:81-109.

Espejo-Serna A., López-Ferrari A.R. y Ugarte I.S. 2004. A current estimate of angiosperm diversity in Mexico. Taxon 53:127130.

Firn R. 2010. Natures's Chemicals: The Natural Products that Shaped our World. Oxford University Press, Oxford. 
Franklin J. 2009. Mapping Species Distributions: Spatial Inference and Prediction. Cambridge University Press, Cambridge.

Gómez-Pompa A. 1965. La vegetación de México. Boletín de la Sociedad Botánica de México 29:76-120.

Gurevitch J., Scheiner S.M. y Fox G.A. 2002. The Ecology of Plants. Sinauer Associates, Sunderland.

Ibarra-Manríquez G., Rendón-Sandoval F.G., Cornejo-Tenorio G. y Carrillo-Reyes P. (Aceptado). Lianas of Mexico. Botanical Sciences.

INEGI. Instituto Nacional de Estadística, Geografía e Informática. 2005. Carta de uso de suelo y vegetación. Escala 1:250 000, Serie III. Intituto Nacional de Estadística, Geografía e Informática, Aguascalientes.

Krebs C.J. 1978. Ecology: The Experimental Analysis of Distribution and Abundance. $2^{\mathrm{a}}$ ed. Harper \& Row, Nueva York.

Krebs J.R. 1992. Evolution and Biodiversity: The New Taxonomy. The report of the Committee setup by The Natural Environment Research Council, Swindon.

Leakey R.E. y Lewin R. 1996. The Sixth Extinction: Patterns of Life and the Future of Humankind. Anchor Books, Nueva York.

Llorente-Bousquets J. y Ocegueda S. 2008. Estado del conocimiento de la biota. En: Comisión Nacional para el Conocimiento y Uso de la Biodiversidad. Capital Natural de México. Vol. I: Conocimiento Actual de la Biodiversidad, pp. 283-322, Comisión Nacional para el Conocimiento y Uso de la Biodiversidad, México, D.F.

Magallón S., Pennington R.T., Villaseñor J.L., Rodríguez P., Honorio C.E.N., Foster R., Harris D. y Wieringa J. 2014. The influence of regional history and ecological affinity in the angiosperm composition of Mexican lowland tropical rainforests. En: Stevens W.D., Montiel O.M. y Raven P. Eds. Paleobotany and Biogeography, pp. 287-325, Missouri Botanical Garden Press, San Luis.

Margules C.R. y Pressey R.L. 2000. Systematic conservation planning. Nature 405:243-253.

Maxted N. 1992. Towards defining a taxonomic revision methodology. Taxon 41:653-660.

McGinley R.J. 1993. Where's the management in collections management? Planning for improved care, greater use, and growth of collections. En: Rose C.L., Williams S.L. y Gisbert J. Eds. The International Symposium and First World Congress on the Preservation and Conservation of Natural History Collections: Current Issues, Initiatives, and Future Directions for the Preservation and Conservation on Natural History Collections, pp. 309-338, Congress Book, Vol. 3. Consejería de Educación y Cultura, Comunidad de Madrid y Dirección General de Bellas Artes y Archivos, Ministerio de Cultura, Madrid.

McNeill J., Barrie F.R., Burdet H.M., Demoulin V., Hawksworth D.L., Marhold K., Nicolson D.H., Prado J., Silva P.C., Skog J.E., Wiersema J.H. y Turland N.J. Eds. 2006. International Code of Botanical Nomenclature (Vienna Code). Adopted by the Seventeenth International Botanical Congress Vienna, Austria, July 2005. Regnum Vegetabile 146:1-568.

Miranda F. y Hernández-X. E. 1963. Los tipos de vegetación de México y su clasificación. Boletín de la Sociedad Botánica de México 28:29-179.

Mittermeier R.A., Turner W.R., Larsen F.W., Brooks T.M. y Gascon C. 2011. Global biodiversity conservation: the critical role of hotspots. En: Zachos F.E. y Habel J.C. Eds. Biodiversity
Hotspots: Distribution and Protection of Conservation Priority Areas, pp. 3-22, Springer-Verlag, Berlín.

Myers N. 1988. Threatened biotas: "hotspots" in tropical forests. Environmentalist 8:187-208.

Myers N., Mittermeier R.A., Mittermeier C.G., da Fonseca G.A.B. y Kent J. 2000. Biodiversity hotspots for conservation priorities. Nature 403:853-858.

Olson D.M., Dinerstei E., Wikramanayake E.D., Burgess N.D., Powell G.V.N., Underwood E.C., D'Amico J.A., Itoua I., Strand H.E., Morrison J.C., Loucks C.J., Allnutt T.F., Ricketts T.H., Kura Y., Lamoreux J.F., Wettengel W.W., Hedao P. y Kassem K.R. 2001. Terrestrial ecoregions of the world: a new map of life on Earth. BioScience 51:933-938.

Pennington T.D. y Sarukhán J. 1968. Árboles Tropicales de México. Instituto Nacional de Investigaciones Forestales, Organización de las Naciones Unidas para la Agricultura y la Alimentación, México, D.F.

Pennington R.T., Cronk Q.C.B. y Richardson J.A. 2004. Introduction and synthesis: plant phylogeny and the origin of major biomes. Philosophical Transactions of the Royal Society of London B 359:1455-1464.

Peterson A.T., Soberón J., Pearson R.G., Anderson R.P., MartínezMeyer E., Nakamura M. y Bastos A.M. 2011. Ecological Niches and Geographic Distributions. Monographs in Population Biology 49. Princeton University Press, Princeton.

Pimm S.L., Russell G.J., Gittleman J.L. y Brooks T.M. 1995. The future of Biodiversity. Science 269:347-350.

Richardson D.M. y Whittaker R.J. 2010. Conservation biogeography - foundations, concepts and challenges. Diversity and Distributions 16:313-320.

Rzedowski J. 1978. Vegetación de México. Limusa, México, D.F.

Rzedowski J. 1991. Diversidad y orígenes de la flora fanerogámica de México. Acta Botanica Mexicana 14:3-21.

Sánchez-Ramos G. y Dirzo R. 2014. El bosque mesófilo de montaña: un ecosistema prioritario amenazado. En: Gual-Díaz M. y Rendón-Correa A. Comps. Bosques Mesófilos de Montaña en México: Diversidad, Ecología y Manejo, pp. 109-139, Comisión Nacional para el Conocimiento y Uso de la Biodiversidad, México, D.F.

Sarukhán J. y Dirzo R. 1992. México ante los Retos de la Biodiversidad. Comisión Nacional para el Conocimiento y Uso de la Biodiversidad, Mexico, D.F.

Soberón J. y Peterson A.T. 2004. Biodiversity informatics: managing and applying primary biodiversity data. Philosophical Transactions of the Royal Society of London B 359:689-698.

Suarez A.V. y Tsutsui N.D. 2004. The value of museum collections for research and society. BioScience 54:66-74.

Systematics Agenda 2000. 1994. Charting the Biosphere: A Global Initiative to Discover, Describe and Classify the World's Species. Technical report. American Museum of Natural History, American Society of Plant Taxonomy, Society of Systematic Biologists, The Willi Hennig Society, Nueva York.

Toledo V.M. 1994. La diversidad biológica de México: nuevos retos para la investigación en los noventas. Revista Ciencias 34:43-59.

Vane-Wright R.I., Humphries C.J. y Williams P.H. 1991. What to protect? - Systematics and the agony of choice. Biological Conservation 55:235-254.

Villaseñor J.L. 2003. Diversidad y distribución de las Magnoliophyta de México. Interciencia 28:160-167. 
Villaseñor J.L. y Murguía-Romero M. 1992. La computadora en la identificación botánica: una grata experiencia. Ciencia y Desarrollo (México) 18:130-137.

Villaseñor J.L. y Ortiz E. 2014. Biodiversidad de las plantas con flores (División Magnoliophyta) en México. Revista Mexicana de Biodiversidad 85(Supl.):S134-S142.

Villaseñor J.L., Ortiz E. y Redonda-Martínez R. 2008. Catálogo de Autores de Plantas Vasculares de México. $2^{\mathrm{a}}$ ed. Instituto de Biología, Universidad Nacional Autónoma de México, Comisión Nacional para el Conocimiento y Uso de la Biodiversidad, México, D.F.

Recibido: 20 de noviembre de 2014

Aceptado: 7 de enero de 2015
Villaseñor J.L., Meave J.A., Ortíz E. e Ibarra-Manríquez G. 2003. Biogeografía y conservación de los bosques tropicales húmedos de México. En: Morrone J.J. y Llorente B.J. Eds. Una Perspectiva Latinoamericana de la Biogeografía, pp. 209-216, Comisión Nacional para el Conocimiento y Uso de la Biodiversidad, Universidad Nacional Autónoma de México, México, D.F.

von Reis Altschul S. 1977. Exploring the herbarium. Scientific American 236:96-104.

Watson M. 1997. On revising a genus. Plant Talk 10:31-34. 